\title{
Translational Research in the Neurobiological Mechanisms of Alcohol and Substance Use Disorders
}

\author{
Carolina L. Haass-Koffler ${ }^{1,2,3}$ (D) - Jesse R. Schank ${ }^{4}$ \\ Published online: 21 January 2020 \\ (C) The American Society for Experimental NeuroTherapeutics, Inc. 2020
}

Alcohol and substance use disorders, as many mental illnesses, are associated with increased mortality in our society [1-3]. While many advances have been made in the field of basic neuroscience, very few of these discoveries have been effectively translated into clinically useful applications to treat this devastating disorder [4]. Furthermore, the limited advances in the development and approval of new medications for treating alcohol and substance use disorders have highlighted the importance of developing more personalized treatment approaches [5]. As such, research in the area of alcohol and substance use disorder treatment, pharmacological or otherwise, is of critical importance.

In this issue of Neurotherapeutics, which focuses on evaluating current translational research into neurobiological targets in alcohol and substance use disorder treatment, an international group of experts provide a comprehensive review of the scientific literature in specific areas of research. This effort will help identify and expand research investigation opportunities. The main goal of this issue is to combine preclinical and clinical neuroscience research expertise from scientists and clinicians to reduce the knowledge gap, identify and describe novel treatment strategies, and improve translational efforts to

Carolina L. Haass-Koffler

carolina_haass-koffler@brown.edu

1 Center for Alcohol and Addiction Studies, Department of Psychiatry and Human Behavior, Warren Alpert Medical School, Providence, USA

2 Department of Behavioral and Social Sciences, School of Public Health, Brown University, Providence, RI, USA

3 Section on Clinical Psychoneuroendocrinology and Neuropsychopharmacology, National Institute on Drug Abuse Intramural Research Program and National Institute on Alcohol Abuse and Alcoholism Division of Intramural Clinical and Biological Research, National Institutes of Health, Bethesda, MD, USA

4 Department of Physiology and Pharmacology, University of Georgia, Athens, Georgia advance the downstream recovery pathway in individuals who suffer from these disorders.

In a thorough review, Hamada and Lasek describe the role of receptor tyrosine kinases (RTKs) in the development and maintenance of alcohol use disorder (AUD) [6]. These receptors, which respond to neurotrophic growth factors and other related molecules, mediate complex alcohol-seeking behaviors. RTKs are active in the regulation of alcohol seeking in specific regions of the brain that are critical nodes of the addiction and motivation circuitry, including the dorsal striatum (tropomyosin-related kinase B (TrkB) and fibroblast growth factor receptor) and the ventral tegmental area (RET protooncogene/glial-cell-line-derived neurotrophic factor and anaplastic lymphoma kinase). Of high translational value, these receptors can be directly manipulated pharmacologically using existing compounds. As highlighted by Hamada and Lasek, several small molecule antagonists, initially developed for the treatment of cancer, are available and can be investigated as potential treatments for alcohol and substance use disorders. TRKs are also represented in an original research article included in this issue. The Gourley Laboratory has shown over the last several years that chronic cocaine administration can induce habit formation. Here, they demonstrate in Pitts et al. that habit formation that is induced by adolescent cocaine exposure can be reversed by stimulating TrkB/brainderived neurotrophic factor (BDNF) signaling in the orbitofrontal cortex [7]. Intriguingly, this effect can be mimicked by 3,4-methylenedioxy-methamphetamine (MDMA) administration, which stimulates BDNF in the orbitofrontal cortex. Another class of proteins for which many available antagonists exist are G protein-coupled receptors (GPCR). In their review, Neasta et al. focus primarily on the approach of genetic deletion or mutation of GPCR function in preclinical animal models [8]. This review covers a wide array of examples within this receptor class and the authors highlight the central role of these receptors in drug and alcohol seeking, providing support for specific candidate targets in drug development. 
In the review by LeCoq et al., the authors discuss in extensive detail the influence of context on drug-seeking behaviors [9]. These contextual factors can take a number of forms including physical environment, social conditions, or interoceptive states induced by drug intake and can significantly influence consumption, subjective drug effects, relapse-like behavior, and drug craving. The authors present translational paradigms in which context can be considered within the framework of major treatment strategies.

Despite high mortality rates due to excessive alcohol consumption and opioid overdose, medications for the treatment of alcohol and opioid use disorder have seen limited use in the USA; for example, less than $10 \%$ of patients with AUD receive any form of treatment [10]. In this issue, Abraham et al. provide a comprehensive overview of medications to treat alcohol and opioid use disorder in clinical practice. The authors suggest that promising strategies to increase availability of alcohol and opioid use disorder medications include incorporating substance use disorder training into healthcare education programs and reducing the stigma surrounding the use of these medications, and that these strategies are also critical to developing novel medications for the treatment of substance use disorder [11].

To facilitate the discovery of possible targets to develop effective treatments for AUD, Oberlin et al. discuss the importance of understanding the interaction between reactivity to alcohol-associated stimuli and compromised executive function, which are hallmarks of AUD [12]. The authors review and identify brain sites of action for these two categories of potential therapies. They show that interventions in the domains of delay discounting and cue reactivity alter brain activity in the ventromedial prefrontal cortex for both domains, and the posterior cingulate/precuneus for delay of gratification. These findings could facilitate targeting and development of new interventions, and ultimately treatments of AUD.

Within the field of behavioral economics, Aston and Meshesha reviewed the marijuana purchase task (MPT), a behavioral economic measure of cannabis demand (i.e., value) [13]. The MPT presents unique caveats compared with the similar alcohol purchase task due to its diversified illicit status. The authors discuss in this review the complex aspect of utilizing MPT in a research setting. Similarly, in the cue reactivity context, Goodyear and Haass-Koffler highlight that while measures of alcohol cue reactivity are widely developed in the alcohol research field, the assessment of cue reactivity to evaluate craving for opioids is relatively limited [14]. The authors discuss some of the major challenges researchers are faced with when assessing opioid craving in clinical research, including the wide range of available assessments that have been developed for measuring craving, establishing the methodological point of departure for designing a study with craving, participant conditions/characteristics which can influence the degree or frequency of opioid craving experienced, and contextual conditions that affect opioid cravings such as a stressful environment that may alter cue saliency.

Targets for the development of medications for alcohol and substance use disorders do not necessarily need to be evaluated using novel pharmacophores, rather potential new treatments can be probed by using medications that are already FDA-approved for different medical indications. In this issue in Neurotherapeutics, Kashyap et al. illustrate that the inability to sustain abstinence, or to seek treatment due to cognitive deficits such as poor decision-making and impulsivity, is known to cause poor treatment outcomes [15]. The authors provide an evidence-based rationale for intranasal drug delivery as a viable and safe treatment modality to bypass the blood-brain barrier and target insulin to the brain to improve the treatment of addiction. Intranasal insulin with improvement of brain cell energy and glucose metabolism, stress hormone reduction, and improved monoamine transmission may represent a novel approach for treating multiple domains of addiction including memory and impulsivity, and may provide additional benefits to enhance current treatment approaches.

The above areas of research have focused on novel pharmacological targets and manipulation of clinical research paradigms; however, there are also exciting alternative possibilities that need to be evaluated in the treatment options for alcohol and substance use disorders. In this issue, Philip et al. present the advances made using noninvasive brain stimulation, most commonly utilizing technologies including transcranial magnetic stimulation and transcranial direct current stimulation, as promising potential treatments for alcohol use disorder [16]. The authors describe the underlying principles behind these technologies, summarize randomized controlled trials, and provide suggestions for future areas of research.

Adopting the Institute of Medicine (IOM) nomenclature to describe where research sits on the translational science spectrum, the papers above deal with preclinical studies (T0), early human laboratory/experimental medicine studies, and earlyphase clinical trials (T1-T2). As treatments hopefully develop to larger samples and to clinical practice (T3-T4), one new challenge emerges in their use in patients who often present with mental health and/or medical comorbidities. An example is represented by AUD patients with alcoholic liver disease (ALD). Addolorato et al. review progress made in the diagnosis and management of AUD in patients with liver disease, which is comprised by several forms ranging from relatively mild and reversible steatosis (fatty liver) and alcoholic hepatitis, to fibrosis, and finally cirrhosis and hepatic failure [17]. Except for baclofen, pharmacological treatments of AUD have not been formally tested in patients with advanced liver disease, and the implementation of psychological approaches is limited in AUD patients and even more in AUD patients with ALD. 
All of the research and treatment topics presented in this issue of Neurotherapeutics focus on strategies that may improve therapies for the treatment of alcohol and substance use disorders and demonstrate that there is a significant future for the integration of research paradigms that can reduce the gap in translating research from basic neuroscience to clinical therapeutic outcomes. These novel treatment strategies may take the form of newly developed pharmacological compounds, the repurposing of already established drugs which have been developed for other indications, or the implementation of novel brain stimulation techniques, among others. Important factors to consider in this development are highlighted in this issue of the journal, including the effective utilization of approved medications and consideration of other existing comorbidities in the management of this population.

Funding Information Dr. Haass-Koffler is supported by the National Institute on Alcohol Abuse and Alcoholism (K01 AA023867; R01 AA026589; R01 AA027760) and by the National Institute of General Medical Sciences (NIGMS), Center of Biomedical Research Excellence (COBRE, P20 GM130414). Dr. Schank is supported by the National Institute on Alcohol Abuse and Alcoholism (R01 AA026362).

\section{Compliance with Ethical Standards}

Conflict of Interest The authors report no biomedical financial interests or potential conflicts of interest.

Required Author Forms Disclosure forms provided by the authors are available with the online version of this article.

\section{References}

1. Green B, Young R, Kavanagh D. Cannabis use and misuse prevalence among people with psychosis. The British Journal of Psychiatry. 2005;187(4):306-13.

2. Koskinen J, Löhönen J, Koponen H, Isohanni M, Miettunen J. Prevalence of alcohol use disorders in schizophrenia-a systematic review and meta-analysis. Acta Psychiatrica Scandinavica. 2009;120(2):85-96.

3. Regier DA, Farmer ME, Rae DS, Locke BZ, Keith SJ, Judd LL et al. Comorbidity of mental disorders with alcohol and other drug abuse: results from the Epidemiologic Catchment Area (ECA) study. Jama. 1990;264(19):2511-8.

4. Heilig M, Sommer WH, Spanagel R. The need for treatment responsive translational biomarkers in alcoholism research.
Translational Neuropsychopharmacology. Springer; 2015. p. 15171.

5. Leggio L, Falk DE, Ryan ML, Fertig J, Litten RZ. Medication Development for Alcohol Use Disorder: A Focus on Clinical Studies. Handb Exp Pharmacol. 2019. doi:https://doi.org/10.1007/ $1642019 \_295$.

6. Hamada K, Lasek AW. Receptor Tyrosine Kinases as Therapeutic Targets for Alcohol Use Disorder. Neurotherapeutics. 2019. doi: https://doi.org/10.1007/s13311-019-00795-4.

7. Pitts EG, Barfield ET, Woon EP, Gourley SL. Action-Outcome Expectancies Require Orbitofrontal Neurotrophin Systems in Naive and Cocaine-Exposed Mice. Neurotherapeutics. 2019. doi: https://doi.org/10.1007/s13311-019-00752-1.

8. Neasta J, Darcq E, Jeanblanc J, Carnicella S, Ben Hamida S. GPCR and Alcohol-Related Behaviors in Genetically Modified Mice. Neurotherapeutics. 2020. https://doi.org/10.1007/s13311-01900828-y.

9. LeCocq MR, Randall PA, Besheer J, Chaudhri N. Considering Drug-Associated Contexts in Substance Use Disorders and Treatment Development. Neurotherapeutics. 2020. doi:https://doi. org/10.1007/s13311-019-00824-2.

10. Abuse S, Administration MHS. Results from the 2013 National Survey on Drug Use and Health: Summary of national findings. NSDUH Series H-48, HHS Publication No (SMA) 14-4863. 2014.

11. Witkiewitz K, Litten RZ, Leggio L. Advances in the science and treatment of alcohol use disorder Sci Adv. 2019;5(9):eaax4043. doi: https://doi.org/10.1126/sciadv.aax4043.

12. Oberlin BG, Shen YI, Kareken DA. Alcohol Use Disorder Interventions Targeting Brain Sites for Both Conditioned Reward and Delayed Gratification. Neurotherapeutics. 2019. doi:https:// doi.org/10.1007/s13311-019-00817-1.

13. Aston ER, Meshesha LZ. Assessing Cannabis Demand: A Comprehensive Review of the Marijuana Purchase Task. Neurotherapeutics. 2020. https://doi.org/10.1007/s13311-01900819-z.

14. Goodyear K, Haass-Koffler CL. Opioid Craving in Human Laboratory Settings: a Review of the Challenges and Limitations. Neurotherapeutics. 2019. doi:https://doi.org/10.1007/s13311-01900791-8.

15. Kashyap B, Hanson LR, Frey Ii WH. Intranasal Insulin: a Treatment Strategy for Addiction. Neurotherapeutics. 2020. doi: https://doi.org/10.1007/s13311-019-00822-4.

16. Philip NS, Sorensen DO, McCalley DM, Hanlon CA. Non-invasive Brain Stimulation for Alcohol Use Disorders: State of the Art and Future Directions. Neurotherapeutics. 2019. doi:https://doi.org/10. 1007/s13311-019-00780-x.

17. Addolorato G, Vassallo GA, Mirijello A, Gasbarrini A. Diagnosis and Management of Alcohol Use Disorder in Patients with Liver Disease: Lights and Shadows. Neurotherapeutics. 2019. doi:https:// doi.org/10.1007/s13311-019-00802-8.

Publisher's Note Springer Nature remains neutral with regard to jurisdictional claims in published maps and institutional affiliations. 